\title{
Expression of tpo mRNA in thyroid tumors: quantitiative PCR analysis and correlation with alterations of ret, Braf, ras and pax 8 genes
}

\author{
J Di Cristofaro ${ }^{1}, M$ Silvy $^{2}$, A Lanteaume ${ }^{3}$, M Marcy $^{4}$, P Carayon $^{1}$ and \\ C De Micco ${ }^{1,4}$
}

\begin{abstract}
${ }^{1}$ Institut National de la Santé et de la Recherche Médicale (U555), IPHM, ${ }^{2}$ Laboratoire de Biochimie et Biologie Moléculaire, ERT-MEIDIA, IFR Jean Roche, ${ }^{3}$ UPRES EA 3287 Pathologie respiratoire liée à l'environnement and ${ }^{4}$ Laboratoire de Pathologie, Faculty of Medicine, Mediterranean University, 27 Bd Jean Moulin, 13385 Marseilles cedex 5, 13385 France

(Requests for offprints should be addressed to J Di Cristofaro; Email: judicristofaro@ hotmail.com)
\end{abstract}

\begin{abstract}
Immunocytochemistry (ICC) of thyroid peroxidase (TPO) using the monoclonal antibody MoAb47 has been used as malignancy marker on thyroid fine needle aspiration. However, little is known about the fate of TPO in thyroid carcinoma. We performed a qualitative PCR (Q-PCR) analysis to measure the expression of variants of tpo mRNA in 13 normal tissue samples, 30 benign tumors (BT), 21 follicular carcinomas (FC), 20 classical papillary carcinomas (PCC), 12 follicular variants of papillary carcinomas (PCfv) and nine oncocytic carcinomas (OC). We also studied mutations involving the ras, Braf, ret or pax8 genes. Results of Q-PCR were closely correlated with those of ICC $(P<0.0001 ; \mathrm{R}=0.59)$ and showed that overall tpo expression was lower in all carcinomas than in normal and BT $(P<0.05)$. The ratio tpo2 or tpo3 to tpo1 was inversed in follicular tumors. Genetic mutations were observed in $90 \%$ of PCc, $61.9 \%$ of FC, $41.7 \%$ of PCfv, $0 \%$ of OC and $10 \%$ in BT. pax8-ppar $\gamma 1$ rearrangement was correlated with qualitative changes in tpo mRNA $(P<0.01)$. These results confirmed the decrease of TPO expression in $97 \%$ of thyroid carcinomas regardless of histological type and the overexpression of shorter splice variants in follicular tumors. Both reduction in quantity of TPO and impairment of its maturation process could account for the atypical immunohistochemical reaction of MoAb47 with TPO.
\end{abstract}

Endocrine-Related Cancer (2006) 13 485-495

\section{Introduction}

Thyroid peroxidase (TPO) is an essential membranebound enzyme in thyroid hormone synthesis. The full-length transcript codes for the 933 amino acid TPO1 protein (Kimura et al. 1987). Differential splicing produces shorter transcripts: tpo2, tpo3 $\left(t p o_{\text {zanelli }}\right)$, tpo4 and tpo5 lacking exon 10, 16, 14 and 8 respectively (Kimura et al. 1987, Zanelli et al. 1990, Elisei et al. 1991, Ferrand et al. 2003). Variants spliced on several exons have also been described: tpo2/3 (10 and 16), tpo2/4 (10 and 14) and tpo6 (10, 12-14 and 16) (Ferrand et al. 2003).

In 1991 we discovered that the anti-TPO monoclonal antibody MoAb47 reacted with less than
$30 \%$ of thyroid carcinomas (De Micco et al. 1991). When present in malignant cells, immunocytochemical (ICC) staining showed an abnormal pattern. Because these differences were so pronounced in comparison with other anti-TPO antibodies (De Micco et al. 1994a, Mizukami et al. 1994), MoAb47 was investigated as a malignancy marker on thyroid fine needle aspiration (FNA) samples. Several independent studies reached similar conclusions. The sensitivity and specificity of this method was close to $100 \%$ in initial short series (De Micco et al. 1994a, Christensen et al. 2000) and around 98\% and $80 \%$ respectively in larger series (Faroux et al. 1997, De Micco et al. 1999). Based on these findings, staining with MoAb47 has been routinely used in several 
Table 1 Relationship between mean tpo mRNA expression (measured by Q-PCR) and pTNM tumor stage

\begin{tabular}{|c|c|c|c|c|c|c|c|c|c|c|c|c|}
\hline \multirow[b]{2}{*}{ Tumor } & \multicolumn{2}{|c|}{ pT1 } & \multicolumn{2}{|c|}{ pT2 } & \multicolumn{2}{|c|}{ pT3 } & \multicolumn{2}{|c|}{ pT4 } & \multicolumn{2}{|c|}{ pNO } & \multicolumn{2}{|c|}{ pN1 } \\
\hline & No. & tpo & No. & tpo & No. & tpo & No. & tpo & No. & tpo & No. & tpo \\
\hline PCc & 7 & 4.59 & 1 & 0.44 & 11 & 5.75 & 1 & 2.82 & 6 & 5.22 & 11 & 4.91 \\
\hline PCfv & 8 & 24.44 & 4 & 29.27 & 0 & & 0 & & 11 & 28.3 & 1 & 1.32 \\
\hline FCmi & 1 & 67.36 & 9 & 55.83 & 1 & 96.93 & 0 & & $x$ & & $x$ & \\
\hline FCwi & 1 & 55.67 & 1 & 9.12 & 7 & 37.04 & 1 & 47.14 & $x$ & & $x$ & \\
\hline OC & 2 & 14.11 & 4 & 34.57 & 2 & 39.02 & 1 & 83.51 & $x$ & & $x$ & \\
\hline
\end{tabular}

$\mathrm{x}$, node status not determined (no surgery on nodes)

laboratories and has proven to be particularly useful in the 'grey area' of follicular tumors (De Micco et al. 1994b, 1999).

The mechanisms underlying TPO anomalies in thyroid tumors remain obscure. Northern blot analysis of tpo mRNA in carcinoma has yielded discrepant results (Hoang-Vu et al. 1992, Elisei et al. 1994) and the hypothesis that an immunological alteration accounts for the variable affinity of TPO for different antibodies (Mizukami et al. 1994, Tanaka et al. 1996) has been questioned (Czarnocka et al. 2001). In pilot studies using immunohistochemistry and in situ hybridization, we observed more heterogeneity in TPO and tpo mRNA in malignant tumors (De Micco et al. 2000). RT-PCR showed that modifications of alternative splicing could account for these variations (Ferrand et al. 2001, Le Fourn et al. 2004).

In this study we measured the expression of complete and spliced variants of tpo mRNA in thyroid tumors using quantitative PCR (Q-PCR). We also studied alterations involving the ras, Braf, ret, or pax8 genes since they might be correlated with the modification in tpo mRNA expression.

\section{Materials and methods}

\section{Patients and samples}

Patients were treated in the Department of Endocrine Surgery at the Timone University Hospital in Marseilles. Patient work-up included FNA biopsy. Tissues taken during surgery were kept frozen. Histological diagnosis was established according to the World Health Organization classification (De Lellis et al. 2004). Pieces of normal tissues $(\mathrm{NT}=13)$ were taken near colloid nodules $(\mathrm{CN})$. The benign tumors $(\mathrm{BT})(n=30)$ included seven $\mathrm{CN}$ and 23 follicular adenomas (FA). The sex ratio (women/men) was $1.31 / 1$ and the mean age was 50.0 years (range, 23-74 years). The carcinomas $(n=62)$ included 11 minimally invasive follicular carcinomas (FCmi), ten widely invasive follicular carcinomas (FCwi), 20 classical papillary carcinomas (PCc) (13 with purely papillary architecture and seven with mixed, papillary and follicular architecture), 12 follicular variants of papillary carcinomas (PCfv) and nine oncocytic carcinomas (OC). The sex ratio (women/men) was $2.44 / 1$ and the mean age was 46.4 years (range, 16-79 years). Tumor stage according to Pathological Tumor Node Metastasis (pTNM) classification is shown in Table 1 . The study protocol was approved by the Clinical Research Committee of Marseilles Public Hospital System. Informed individual consent to use residual pieces of tissues for research purpose was obtained.

\section{ICC}

ICC for TPO was routinely performed concurrently with Giemsa staining on one or two slides of FNA biopsy. Smears for ICC were air-dried and immediately stored at $4{ }^{\circ} \mathrm{C}$ until staining. ICC consisted of overnight incubation at $4{ }^{\circ} \mathrm{C}$ with MoAb47 $(1 / 100)$ (DAKO, Glostrup, Denmark), incubation with a streptavidin-biotin-peroxidase kit (LSAB; DAKO) and revelation of peroxidase activity with a $3,3^{\prime}$ diaminobenzidine $-\mathrm{H}_{2} \mathrm{O}_{2}$ kit. FNA samples from surgically removed benign tissue was used as a positive control. The primary antibody was omitted from negative controls. The percentage of positive cells was recorded, excluding cells exhibiting abnormal staining with fading of perinuclear staining (De Micco et al. 1994a).

\section{RNA isolation and cDNA synthesis}

RNA isolation was performed using Trizol (Invitrogen, Cergy-Pontoise, France). RNA was extracted with $200 \mu$ l chloroform and precipitated with $350 \mu 1$ 


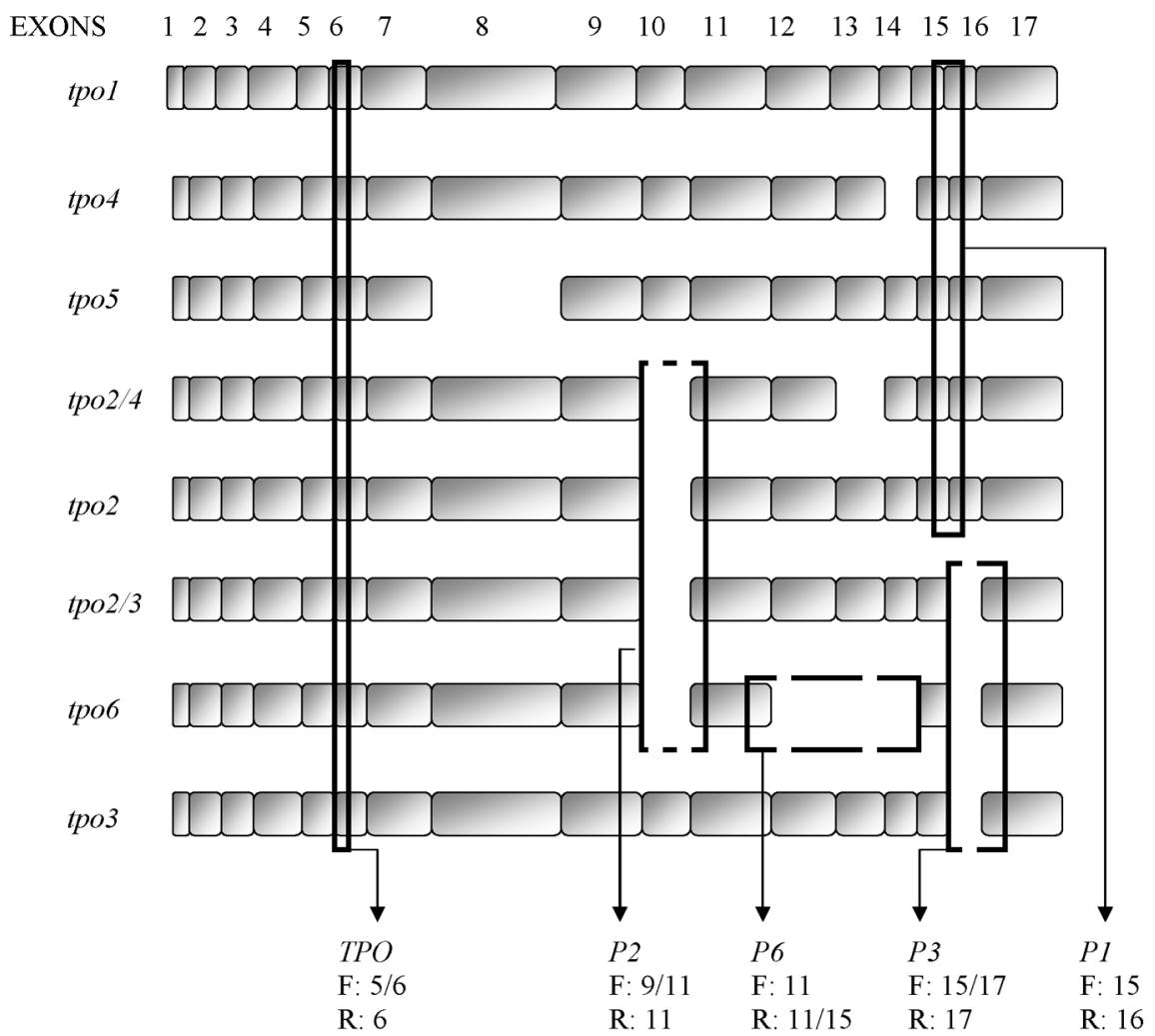

Figure 1 Schematic representation of tpo variants showing deleted exons. Black boxes indicate the positions of Q-PCR primer pairs $P 1, P 2, P 3, P 6$ and TPO. F, forward; R, reverse.

isopropanol overnight at $-207^{\circ} \mathrm{C}$. After centrifugation and washing, pellets were diluted in $20 \mu \mathrm{l}$ diethyl pyrocarbonate-treated water. RNA concentration was estimated with an optical density absorption setting of $260 \mathrm{~nm}$. One microgram of RNA was reverse-transcribed in a volume of $20 \mu \mathrm{l}$ using random priming and Superscript II (Invitrogen).

\section{Q-PCR}

A two-step relative method was used to quantify tpo mRNA. First, expression of total tpo mRNA in each sample was normalized to expression of the tata box binding protein gene $(t b p)$ and compared with the median of values from NT (Picard et al. 2006). Secondly, the ratios of tpo variants to total tpo were determined in each sample.

Primers were designed using Primer Express software (Fig. 1) (Applied Biosystems, Courtabœuf, France). Their characteristics are given in Table 2. Analysis of total tpo mRNA expression was performed with TPO primers amplifying a sequence in exons 5-6. Primers spanning the banks of deleted exons were designed for separate quantification of tpo variants (Vandenbroucke et al. 2001). The $P 1$ primer pair amplified all variants, except those lacking exon 16 (tpo1, tpo2, tpo4, tpo5 and tpo2/4). The $P 2$ primer pair amplified variants lacking exon 10 (tpo2, tpo6, tpo2/3 and tpo2/4). The $P 3$ primer pair amplified variants lacking exon 16 (tpo3, tpo6 and tpo2/3). The P6 primer pair amplified only tpo6.

Q-PCR experiments were performed on an ABI Prism-7700 system (Perkin-Elmer/Applied Biosystems). The amplification reaction was performed using $1 \times$ SYBR GreenI PCR Mastermix (Eurogentec, Angers, France), $5 \mu \mathrm{cDNA}$ and $300 \mathrm{nM}$ of each primer. The PCR program consisted of an initial step at $95^{\circ} \mathrm{C}$ for 5 min followed by a 40 -cycle melting step at $95^{\circ} \mathrm{C}$ for $15 \mathrm{~s}$ and an annealingelongation step at $59^{\circ} \mathrm{C}$ for $1 \mathrm{~min}$. In each experiment, a standard curve was used to estimate PCR efficiency and dissociation curves were performed to check the absence of non-specific amplification and primer-dimer formation. PCR products were also checked on agarose gel. Non-template controls were systematically performed. At least two replicates were performed for each experiment. Reproducibility of Q-PCR experiments for $P 1, P 2$ and $P 6$ was 
Table 2 Characteristics of primers used for PCR and Q-PCR analysis of tpo mRNA variants and oncogenes

\begin{tabular}{|c|c|c|c|c|c|c|}
\hline Primer pair & & Sequence $\left(5^{\prime}-3^{\prime}\right)$ & $\begin{array}{l}\text { Localization } \\
\text { (exon) }\end{array}$ & $\begin{array}{c}\text { Accession } \\
\text { number }\end{array}$ & $\begin{array}{l}\text { Size } \\
\text { (bp) }\end{array}$ & $\begin{array}{l}\mathrm{Tm} \\
\left({ }^{\circ} \mathrm{C}\right)\end{array}$ \\
\hline \multirow[t]{2}{*}{ tbp } & $F$ & CAC GAA CCA CGG CAC TGA TT & 4 & ВТ019657 & 89 & 59 \\
\hline & $R$ & TTT TCT TGC TGC CAG TCT GGA C & 5 & & & 59 \\
\hline \multirow[t]{2}{*}{ TPO } & $F$ & AAC AAC AGA GAC CAC CCC AGA T & $5 / 6$ & BC095448 & 86 & 59 \\
\hline & $R$ & TGA CTG AAG CCG TCC TCA TAG AC & 6 & & & 59 \\
\hline \multirow[t]{2}{*}{$P 1$} & $F$ & CGG AGG CTT CGC AGG TC & 15 & BC095448 & 68 & 59 \\
\hline & $R$ & ACG CAC TGG CAC TAA ATC CAC AC & 16 & & & 59 \\
\hline \multirow[t]{2}{*}{$P 2$} & $F$ & CGT GGA GGT TAC AAT GAG TGG A & $9 / 11$ & BC095448 & 138 & 59 \\
\hline & $R$ & CGA TGT TGT CAG GAT GCT TGT AC & 11 & & & 59 \\
\hline \multirow[t]{2}{*}{ P3 } & $F$ & TTT GCA GGT GGA GAG TGC TG & $15 / 17$ & BC095448 & 78 & 59 \\
\hline & $R$ & CTG CCA CTT TGC CCT CAG GA & 17 & & & 59 \\
\hline \multirow[t]{2}{*}{ P6 } & $F$ & GCT GGG AGG CTT AGT TGA AA & 11 & BC095448 & 112 & 59 \\
\hline & $R$ & CCC GGA GTA CCA GTC ACC AT & $11 / 15$ & & & 59 \\
\hline \multirow[t]{2}{*}{ Braf } & $F$ & GCA CAG GGC ATG GAT TAC TT & $13 / 14$ & M95712 & 195 & 59 \\
\hline & $R$ & GAT GAC TTC TGG TGC CAT CC & $15 / 16$ & & & 59 \\
\hline \multirow[t]{2}{*}{ Nras } & $F$ & ATG ACT GAG TAC AAA CTG GT & 1 & X02751 & 288 & 54 \\
\hline & $R$ & GTA GAG GTT AAT ATC CGC AA & 2 & & & 54 \\
\hline \multirow[t]{4}{*}{ pax 8-ppar $\gamma 1$} & $F 1$ & AAC CTC TCG ACT CAC CAG AC & pax8 ex 7 & X69699 & $362 / 68$ & 60 \\
\hline & $F 2$ & CCC TTC AAT GCC TTT CCC C & pax8 ex 8 & & $172 / 67$ & 60 \\
\hline & $F 3$ & CTA TGC CTC CTC TGC CAT C & pax8 ex 9 & & 69 & 60 \\
\hline & $R$ & AGA ATG GCA TCT CTG TGT CAA C & ppar $\gamma$ ex 1 & NM_138712 & & 60 \\
\hline \multirow[t]{2}{*}{ ret/PTC1 } & $F$ & ATT GTC ATC TCG CCG TTC & $H 4$ ex 1 & M31213 & 306 & 54 \\
\hline & $R$ & CTT TCA GCA TCT TCA CGG & ret ex 12 & & & 54 \\
\hline \multirow[t]{2}{*}{ ret/PTC3 } & $F$ & AAG CAA ACC TGC CAG TGG & Ele1 ex 7 & S71225 & 244 & 56 \\
\hline & $R$ & CTT TCA GCA TCT TCA CGG & ret ex 12 & & & 54 \\
\hline
\end{tabular}

$F$, forward; $R$, reverse; ex, exon; Tm, melting temperature.

evaluated in 25 samples by calculating the coefficient of variation $(\mathrm{CV})$ using four measures on each sample. The mean $\mathrm{CV}$ values were $1.7 \%( \pm 0.74)$, $2.5 \%( \pm 1.27)$ and $1.9 \%( \pm 1.04)$ for $P 1, P 2$ and $P 6$ respectively. The $t b p \mathrm{CV}$ for all samples was $3.3 \%$.

\section{Oncogene analysis}

Primers used to amplify ret/papillary thyroid carcinoma (PTC)1, ret/PTC3 (Santoro et al. 2000), exon 15 of Braf (Nikiforova et al. 2004), exons 1 and 2 of pax8/ppar $\gamma 1$ (Nikiforova et al. 2002) and Nras (Vasko et al. 2003) have been described previously. Reactions were performed in $25 \mu$ of a PCR solution containing $4 \mu \mathrm{l}$ cDNA, $5 \mathrm{mM} \mathrm{MgCl}_{2}, 1 \times$ buffer, $4 \mathrm{ng} / \mu \mathrm{l}$ primer and $0.25 \mathrm{U}$ Taq polymerase (Promega, Charbonnières, France). The amplification process included the following steps: denaturation at $94{ }^{\circ} \mathrm{C}$ for $5 \mathrm{~min}$, amplification by 35 cycles of denaturation at $94{ }^{\circ} \mathrm{C}$ for $30 \mathrm{~s}$, hybridization with $\mathrm{Tm}$ primers for $1 \mathrm{~min}$ and elongation at $72{ }^{\circ} \mathrm{C}$ for $1 \mathrm{~min}$. PCR products were visualized by electrophoresis in $1.5 \%$ agarose gel. Products were submitted to direct sequencing (Genome Express, Grenoble, France). Mutations were confirmed by sequencing of antisense strands.

\section{Statistical analysis}

Frequencies of single variables were compared using the $\chi^{2}$ or Fisher exact test. Oncogene mutations and patient age were analysed by Student's $t$-test. After checking for normal distribution of individual tpo mRNA values, mean values were analyzed by ANOVA and the Bonferroni test. $P$ values less than 0.05 were considered as significant. Linear regression was studied to compare Q-PCR and ICC TPO values. All statistical tests were performed using the Statistical Package for Social Sciences (SPSS, Inc., Chicago, IL, USA).

\section{Results}

\section{ICC}

All follicular cells from BT and NT showed diffuse, cytoplasmic labeling of TPO that was more pronounced in the perinuclear area, thus giving a characteristic ring-shaped pattern (Fig. 2). More than $80 \%$ positive cells were found in six of seven $\mathrm{CN}$ and 21 of $23 \mathrm{FA}$ and mean values were significantly higher in FA and $\mathrm{CN}$ than all histological types of carcinoma $(P<0.0001)$ (Table 3$)$. All carcinomas except two FC contained less than $80 \%$ positive 

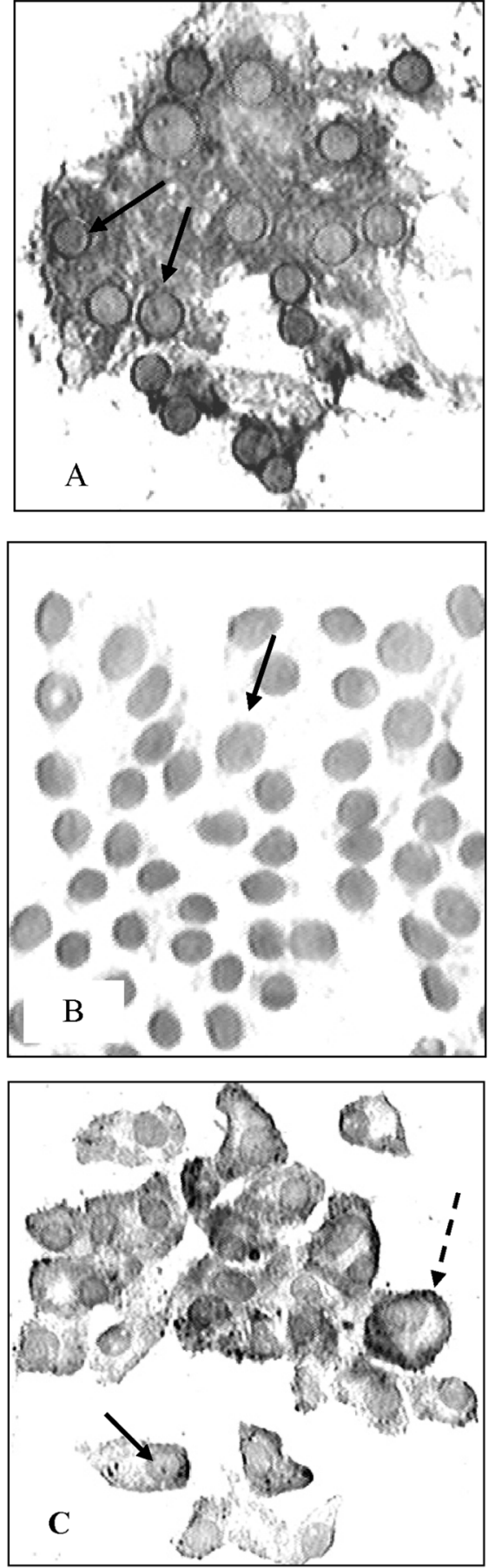

Figure 2 ICC staining of TPO in thyroid cells obtained by FNA $(\times 400)$. (A) FA: a homogeneous dark brown reaction is present in the cytoplasm and the perinuclear area (arrows) in all cells.

(B) Papillary carcinoma: absence of immunostaining in the cytoplasm and around nuclei (arrow shows a nucleus: the light grey color of the nuclei is due to hemotoxylin counterstaining). (C) FC: a heterogeneous coarse granular positive reaction is present in some cells on the edge of the cytoplasm (broken arrow); perinuclear staining is absent (arrow).
Table 3 Analysis of TPO expression by ICC and Q-PCR of overall tpo expression

\begin{tabular}{|c|c|c|c|c|}
\hline \multirow[b]{2}{*}{ Tissue type } & \multicolumn{2}{|c|}{ ICC $^{*}$} & \multicolumn{2}{|c|}{ Q-PCR ${ }^{* *}$} \\
\hline & Mean & Range & Mean & Range \\
\hline NT & 100.0 & $100-100$ & 111.1 & 46.9-221.9 \\
\hline FC & 42.6 & $0-100$ & 49.4 & $0.3-120.1$ \\
\hline $\mathrm{FCmi}$ & 48.2 & $10-80$ & 60.6 & $25.6-120.1$ \\
\hline FCwi & 36.5 & $0-100$ & 37.1 & $0.3-65.5$ \\
\hline PCfv & 30.0 & $0-60$ & 26.0 & $1.3-60.7$ \\
\hline PCc & 9.7 & $0-60$ & 4.9 & $0.1-29.1$ \\
\hline OC & 37.8 & $0-60$ & 36.4 & $2.9-118.1$ \\
\hline BT & 90.6 & $60-100$ & 126.0 & $14.9-456.3$ \\
\hline $\mathrm{FA}$ & 90 & $60-100$ & 111.4 & $17.8-342.3$ \\
\hline $\mathrm{CN}$ & 91.6 & $70-100$ & 173.6 & $14.9-456.3$ \\
\hline
\end{tabular}

${ }^{*}$ Results of ICC on FNA samples are expressed as the percentage of positive cells.

${ }^{* *}$ Results of Q-PCR analysis are expressed as the percentage of the median value found in normal tissues.

cells. TPO expression was very low in PCc with a mean value significantly lower than in $\mathrm{OC}$ and $\mathrm{FC}$ (9.7\% vs 37.8 and $42.9 \% ; P=0.015$ and $P<0.001$ respectively). PCfv (30\%) and OC displayed intermediate mean values between PCc and FC. When present in malignant cells, staining was coarse and located at the periphery of the cytoplasm. The perinuclear ring was missing (Fig. 2).

\section{Q-PCR}

Mean total tpo mRNA was significantly lower in all histological types of carcinomas than in NT $(111.2 \%)$ and BT $(126.0 \%)(P<0.01)$ (Table 3). Expression in PCc was the lowest $(4.9 \%)$, always below $30 \%$ of the normal value (Fig. 3) and significantly lower than in OC and FC $(P=0.015$ and $P<0.001$ respectively). Intermediate values were found in OC $(36.4 \%)$ and PCfv $(26 \%)$. The highest

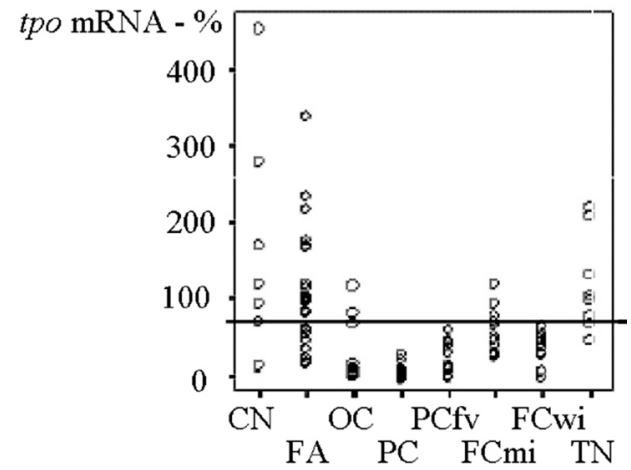

Figure 3 Q-PCR analysis of total tpo expression. Distribution in individual cases. The horizontal line shows the cut-off value (73\%). 
Table 4 Expression of tpo variants amplified with $P 1, P 2, P 3$ and $P 6$ primer pairs. Results are expressed as percentage of total tpo mRNA

\begin{tabular}{lcccc}
\hline Tissue type & $\boldsymbol{P 1}$ & $\boldsymbol{P 2}$ & $\boldsymbol{P 3}$ & $\boldsymbol{P 6}$ \\
\hline NT & $71.0 \pm 14.8$ & $49.9 \pm 8.8$ & $14.8 \pm 4.2$ & $4.5 \pm 4.1$ \\
FC & $89.3 \pm 24.1$ & $84.1 \pm 26.2$ & $20.5 \pm 7.8$ & $3.0 \pm 4.6$ \\
FCmi & $101.4 \pm 23.4$ & $92.9 \pm 19.7$ & $22.9 \pm 6.9$ & $3.5 \pm 5.8$ \\
FCwi & $75.9 \pm 17.5$ & $74.3 \pm 29.8$ & $17.8 \pm 8.1$ & $2.4 \pm 2.8$ \\
PCfv & $87.0 \pm 20.8$ & $72.6 \pm 31.1$ & $18.2 \pm 4.4$ & $3.9 \pm 3.9$ \\
PCC & $84.3 \pm 23.5$ & $74.6 \pm 23.2$ & $15.1 \pm 4.6$ & $6.5 \pm 5.5$ \\
OC & $74.9 \pm 32.4$ & $74.9 \pm 15.7$ & $25.6 \pm 18.1$ & $6.5 \pm 9.0$ \\
BT & $71.2 \pm 46.4$ & $53.1 \pm 30.4$ & $8.3 \pm 7.6$ & $3.9 \pm 3.8$ \\
FA & $70.6 \pm 50.6$ & $47.0 \pm 26.7$ & $8.1 \pm 8.2$ & $3.5 \pm 3.8$ \\
CN & $73.2 \pm 31.8$ & $73.3 \pm 35.1$ & $9.0 \pm 5.6$ & $5.2 \pm 3.7$ \\
\hline
\end{tabular}

values in carcinoma were observed in $\mathrm{FC}(49.3 \%)$. Mean content in FCmi $(60.6 \%)$ was significantly higher than in FCwi $(37.1 \% ; P=0.04)$ and significantly lower than in $\mathrm{CN}$ and NT $(P=0.005$ and $P=0.01$ respectively). Mean FCmi content was also lower than in FA but this difference was not statistically significant $(P=0.6)$. Only one $\mathrm{OC}$ and one FCmi expressed tpo mRNA values in the normal range $(118.1 \%$ and $120.2 \%$ respectively). Mean tpo expression was similar in FA and NT. It was higher in $\mathrm{CN}$ than NT but this difference was not statistically significant $(P=0.09)$.

Q-PCR analysis of tpo mRNA allowed accurate discrimination of $\mathrm{BT}$ and cancer. A receiver operating characteristic (ROC) curve showed that the best tpo mRNA threshold value for discrimination of malignancy was $73 \%$. Values less than $73 \%$ were observed in eight of $23 \mathrm{FA}$ and one of seven $\mathrm{CN}$ and values greater than $73 \%$ were observed in three of 11 FCmi and two of nine OC. Using this cut-off value, the sensitivity and specificity of tpo mRNA Q-PCR analysis for diagnosis of malignancy were 92 and $70 \%$ respectively. However, the mean values of total tpo mRNA were not correlated with the pTNM stage of the tumors (Table 1). Several PCc at stage T1N0 expressed less than $1 \%$ of normal values whereas the two tumors at stage T4 (one FC and one OC) expressed 47.14 and $83.51 \%$ of normal values respectively.

For each sample, we calculated the ratio of variants amplified with the $P 1, P 2, P 3$ and $P 6$ primers to the total tpo amplified with the $T P O$ primers (Table 4).

$P 1$ variant ratios were higher in all malignant tumors than in NT. The mean ratio in FA was close to normal. The highest ratio observed in carcinoma was in $\mathrm{FCmi}$ and was significantly higher than in NT $(P=0.001)$ and than in FCwi $(P=0.01)$.
$P 2$ variant expression in malignant tumors was higher than in NT. The mean ratio in FA was close to normal. Mean ratio in FC was significantly different from NT $(P=0.01)$. FCmi presented the highest mean $P 2$ value that was significantly different from the mean $P 2$ value in NT and BT ( $P=0.003$ and $P=0.001$ respectively). The only FCmi expressing normal total tpo mRNA (120.2\%) also expressed $105.3 \%$ of $P 2$ variant.

All carcinomas had higher mean $P 3$ ratios than NT. BT had a lower mean $P 3$ ratio than NT. Ratios in PCc were close to normal. The highest rates were observed in $\mathrm{FCmi}$ and $\mathrm{OC}$. The mean ratio in FCmi was significantly higher than in NT $(P=0.02)$ and $\mathrm{BT}(P=0.001)$. The ratio in $\mathrm{OC}$ was significantly higher than in both BT $(P=0.001)$ and PCc $(P=0.01)$. The ratio in PCfv was intermediate between the ratios in FC and PCc.

Mean $P 6$ variant expression in relation to NT was higher in OC and PCc and lower in FC, PCfv and BT. PCfv presented intermediate values between FC and PCc. However, the amount of P6 variant was highly variable from case to case and these differences were not significant.

\section{ICC and Q-PCR correlation}

The correlation was determined between TPO expression studied by ICC with MoAb47 and total tpo mRNA expression measured by Q-PCR. A linear regression curve was drawn using data from all samples. Findings showed a highly significant correlation $(P<0.0001)$ with a coefficient $(\mathrm{R})$ equal to 0.59 (Fig. 4).

\section{Oncogene defects}

Oncogene mutations and rearrangements are summarized in Table 5. All Nras mutations occurred 


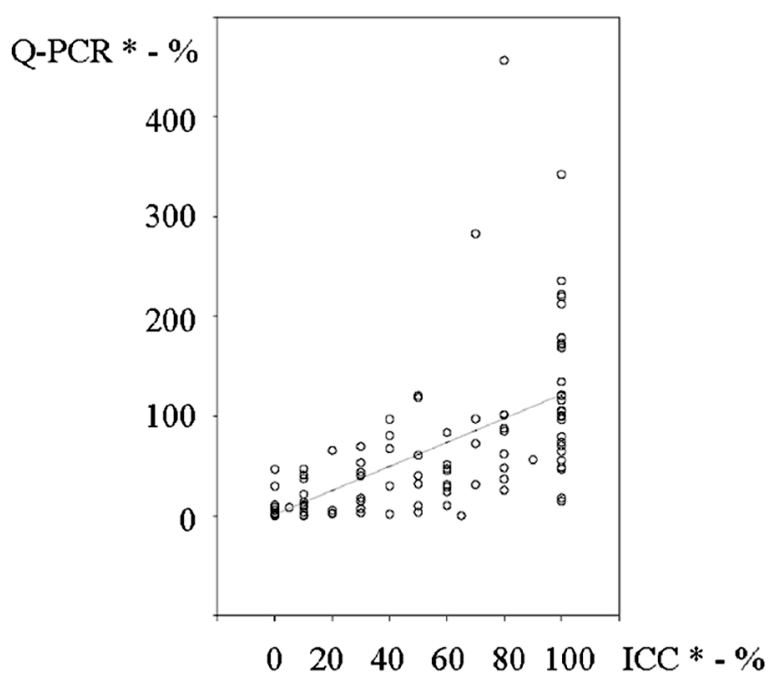

Figure 4 TPO expression: comparison of ICC on FNA and $\mathrm{Q}-\mathrm{PCR}$ analysis by linear regression shows a significant correlation $(\mathrm{R}=0.59$ and $P<0.0001)$. ${ }^{*} \mathrm{ICC}$ of TPO on FNA samples is expressed as the percentage of positive cells. Q-PCR of tpo mRNA is expressed as the percentage of the value in normal tissues.

at codon 61 (exon 2), with equal incidence of transition and transversion. Mutations were present in $38.1 \%$ of FC, $25 \%$ of PCfv, $10 \%$ of TB and no OC. Nras mutation was not correlated with tpo mRNA expression or clinical features, but was significantly more frequent in $\mathrm{FC}$ than in $\mathrm{FA}$ $(P=0.02)$.

pax8/ppar $\gamma 1$ rearrangements were present in $42.8 \%$ of $\mathrm{FC}, 8.3 \%$ of PCfv and no $\mathrm{OC}, \mathrm{CN}$ and FA. Eight out of ten rearrangements involved fusion between exon 9 of pax8 and exon 1 of ppar $\gamma 1$, with or without exon 8 of pax8. Two rearrangements involved fusion between exon 7 of pax 8 and exon 1 of ppar $\gamma 1$.
Patients presenting FC with pax8/ppar $\gamma 1$ were significantly younger than patients presenting FC without the rearrangement (43.3 vs 58.1 years; $P=0.04)$. This difference was also observed in patients presenting $\mathrm{PCfv}$ with or without pax8/ppar $\gamma 1$ (32 vs. 43.8 years; $P=0.04$ ). The incidence of pax8/ppar $\gamma 1$ was higher in FCmi (six of 11) than FCwi (three of ten) but this difference was not statistically significant. pax8/ppar $\gamma 1$ was not correlated with gender.

A significant increase in mean $P 1$ variant expression was found in FC with pax8/ppar $\gamma 1$ as compared with FC without pax8/ppar $\gamma 1$ (104.1 \pm $26.5 \%$ vs $78.1 \pm 15.4 \%, P=0.01)$.

Braf mutations were present in $75 \%$ of PCc and $8.3 \%$ of PCfv $(P<0.001)$. They were never observed in BT and OC. All Braf mutations involved T to A missense transversion at nucleotide 1799. One PCc presented a previously unreported mutation at nucleotide 1799-1800 (TG>AA) that also led to valine-to-glutamate substitution. No K601E mutation was found. Braf mutation was not correlated with gender but it was correlated with older age in patients with PCc (46.3 vs 29.8 years, $P=0.02$ ). It was more frequent in $\mathrm{PCc}$ with pure papillary architecture (12 of 13) than in PCc with mixed papillary and follicular architecture (three of seven), but the difference was not statistically significant. Total tpo mRNA expression was lower in PCc with as opposed to without Braf mutation (3.4\% vs $13.5 \%)$, but this difference was not statistically significant.

A ret/PTC rearrangement was found in five PCc, three PTC1 and three PTC3. One tumor exhibited both PTC1 and PTC3; it was at stage T3N1 with extra-glandular extension and multiple lymph-node metastasis. No other tumor type presented ret/PTC rearrangements. No correlation was found between ret/PTC and tpo expression or clinical features.

Table 5 Clinicopathological data and oncogene defects in 92 thyroid tumors

\begin{tabular}{|c|c|c|c|c|c|c|c|c|}
\hline Histology & $\begin{array}{l}\text { No. of } \\
\text { cases }\end{array}$ & $\begin{array}{l}\text { Female/ } \\
\text { male }\end{array}$ & $\begin{array}{c}\text { Mean age } \\
\text { (range) }\end{array}$ & Nras* & $\begin{array}{c}\operatorname{pax8-} \\
\operatorname{ppar\gamma } 1^{*}\end{array}$ & Braf* & ret $/$ PTC1 $^{*}$ & ret/PTC $3^{*}$ \\
\hline Carcinomas & 62 & & & & & & & \\
\hline PCc & 20 & $2.5 / 1$ & $41(16-70)$ & - & - & $15(75)$ & $3(15)$ & $3(15)$ \\
\hline PCfv & 12 & $1.75 / 1$ & $43(29-77)$ & $3(25.0)$ & 1 (8.3) & 1 (8.3) & 0 & 0 \\
\hline $\mathrm{FCmi}$ & 11 & $2.7 / 1$ & $51(31-73)$ & $4(36.4)$ & $6(54.5)$ & - & - & - \\
\hline FCwi & 10 & $2.3 / 1$ & $53(20-79)$ & $4(40)$ & $3(30)$ & - & - & - \\
\hline OC & 9 & $8 / 1$ & $48(26-71)$ & 0 & 0 & 0 & - & - \\
\hline Benign tumors & 30 & & & $3(10)$ & & & & \\
\hline FA & 23 & $1.55 / 1$ & $53(23-74)$ & $2(8.7)$ & $0 / 9$ & $0 / 9$ & 0/9 & $0 / 9$ \\
\hline $\mathrm{CN}$ & 7 & $0.75 / 1$ & $39(23-58)$ & $1(14.3)$ & $0 / 4$ & $0 / 4$ & $0 / 4$ & $0 / 4$ \\
\hline
\end{tabular}

*Number of mutations or rearrangements (\%). 
Several tumors displayed more than one molecular alteration. Four FC (19\%) exhibited both Nras mutations and pax8/ppar $\gamma 1$ rearrangements. These tumors displayed no special histological or clinical features. Two PCc exhibited both ret/PTC (one PTC1 and one PTC3) and Braf mutations. Both were at stage T3 and were characterized by multiple intra-thyroid tumor foci and extra-glandular extension.

\section{Discussion}

In this study we used Q-PCR to measure total tpo mRNA expression and determine the relative proportion of the main spliced variants in normal and neoplastic thyroid tissue specimens. We also studied the presence ret/PTC rearrangements, pax8/ppar $\gamma 1$ rearrangements, Nras mutations and Braf mutations.

Results of Q-PCR analysis of total tpo mRNA correlated closely with those of ICC using MoAb47 and confirmed a decrease in TPO expression in $97 \%$ of thyroid carcinomas, regardless of histological type. Reduction in TPO expression by malignant thyroid tumors has already been reported but previous studies concerned mainly PC (Hoang$\mathrm{Vu}$ et al. 1992, Tanaka et al. 1996, Lazar et al. 1999, Huang et al. 2001). This reduction stems from a decrease in PAX8 expression and parallels the decrease of many other differentiated functions such as sodium iodide symporter expression (Fabbro et al. 1994, Lazar et al. 1999). Our study also includes a large series of benign and malignant follicular tumors. We found that mean tpo expression was significantly lower in PCc than in NT, FA and FC but also significantly lower in FC, OC and PCfv than in FA. In agreement with Lazar et al. (1999) we found similar tpo expression in FA and NT. Overlapping values were found in some individual cases of FA and FCmi exhibiting unusually low and high levels of tpo expression respectively. This overlapping was consistent with recent data suggesting the existence of an adenoma-carcinoma sequence in which a subset of FA might predispose to malignant transformation (Vasko et al. 2004, Sarquis et al. 2006). The finding that tpo is significantly lower in FCwi than FCmi and higher in PCfv than PCc confirms the strong correlation between tpo expression and follicular differentiation. The correlation with histological differentiation also appears in PCc since tumors with mixed papillary and follicular architecture expressed more TPO than tumors with pure papillary architecture.
Conversely, clinical pTNM staging based mainly on tumor size and extra-thyroid invasiveness has little correlation with tpo expression as long as the tumor tissue remains differentiated. Regarding differentiation, it should be noted that dedifferentiation of FC is often correlated with invasiveness, which would explain why tpo expression was usually lower in highly invasive than encapsulated tumors.

We found that the relative proportion of spliced tpo variants was enhanced in carcinomas, especially $\mathrm{FC}$ and $\mathrm{OC}$. The increase was significant for $P 2$ variants in $\mathrm{FC}$ and $P 3$ variants in OC. Involvement of the whole spectrum of differentiated follicular tumors but not of PCc suggests that disruption of tpo mRNA maturation is a characteristic feature of follicular thyroid carcinogenesis.

The increase in shorter TPO isoforms could account for several features of follicular tumors and explain the variations observed in ICC experiments using various anti-TPO antibodies. Since it has been shown that TPO2 is folded incorrectly, unable to bind to heme and enzymatically inactive (Niccoli et al. 1997), predominance of TPO2 over TPO1 might reduce iodine concentration activity in tumor tissue. In addition, TPO2 and TPO3 have a shorter half-life than TPO 1 ( 3 and $7 \mathrm{~h}$ vs $11 \mathrm{~h}$ respectively), and remain almost exclusively in the intracellular compartment instead of reaching the cell surface (Niccoli et al. 1997, Niccoli-Sire et al. 2001). Immunological profiles cannot explain the differences in reactivity between MoAb47 and other monoclonal antibodies since MoAb47 recognized both TPO2 and TPO3 under experimental conditions (Niccoli et al. 1997, Niccoli-Sire et al. 2001). Rapid turnover of TPO 2 and TPO3 might provide a more plausible explanation for the weak reaction obtained with MoAb47 on malignant follicular tumors. Indeed, a special property of MoAb47 in comparison with other antibodies is to react with a native, unglycosylated TPO, having a short halflife, rather than with mature forms of the protein (Chazenbalk et al. 1993, Fayadat et al. 1998). In NT, the concentration of native TPO and tpo mRNA is higher in the nuclear envelope than in the endoplasmic reticulum (Pohl et al. 1993). In tumor tissue, overproduction of mistargeted variants with a short half-life and the quantitative decrease of TPO expression could explain both the reduction of immunostaining obtained with MoAb47 and its location in the cytoplasm rather than in the perinuclear area. The presence of abnormally located TPO in malignant follicular tumors still expressing a significant amount of the protein 
lowers the risk of overlooking the malignancy with ICC. For this reason, ICC appears to be more accurate for diagnosing malignancy than Q-PCR which only measures quantitative variations of tpo expression.

The high versus low incidence of Braf mutations and ret/PTC respectively found in PCc can be explained by the clinico-pathological features, i.e. patient age over 35 years in most cases, higher frequency of advanced tumor stage and purely papillary histology. These three features have been correlated with Braf mutations and ret/PTC previously (Cohen et al. 2003, Kim et al. 2004, Nikiforova et al. 2004, Trovisco et al. 2004, Di Cristofaro et al. 2005, Xing et al. 2004). The antagonism between oncogene activation of the ERK signaling cascade and expression of differentiated functions by thyroid cells has been confirmed by expression array analysis and may explain the inhibition of tpo expression in PC (Melillo et al. 2005). In our study, all PCc exhibited low tpo expression and PCc harboring Braf ${ }^{T 1799 A}$ mutation were associated with even lower tpo values. This difference was not statistically significant probably because the number of PCc without $\mathrm{Braf}^{\text {T1799A }}$ mutation was too small.

In FC, the rates of pax8-ppar $\gamma 1$ and Nras mutation were similar and within the same range as previously reported (Nikiforova et al. 2002, 2003, Dwight et al. 2003, Vasko et al. 2003). Unlike Nikiforova et al. (2003), we found overlapping in $19 \%$ (four of 21 ) of FC. This suggests that the two events are not mutually exclusive and that concurrence might contribute to the emergence of different phenotypes.

We found that pax8-ppar $\gamma 1$ has a direct impact on tpo maturation since a significant predominance of variants amplified by $P l$ was observed in tumors exhibiting pax8-ppar $\gamma 1$. Expression profile analysis of FC displaying pax8-ppar $\gamma 1$ fusion oncogene demonstrated impairment of ribosomal proteins and of translation-associated genes that could explain disruption of the protein maturation process (Lui et al. 2005). Further study will be needed to determine the exact effect of pax8-ppar $\gamma l$ on tpo mRNA maturation.

Unlike the pax8-ppar $\gamma 1$ rearrangement, ras mutation was not correlated with tpo expression. This unexpected finding supports recent speculation that FC with pax8-ppar $\gamma 1$ and FC with ras mutation constitute distinct biological entities (Nikiforova et al. 2003, Lui et al. 2005). Experimental data in human thyroid epithelial cells show that mutant
RAS induces self-limited proliferation without loss of differentiation (Gire \& Wynford-Thomas 2000). This finding fits well with our observation that Nras mutation has a weak effect on tpo expression and that another more determinant event is necessary to explain its downregulation in FC.

None of the molecular mutations investigated here was found in OC. This suggests that the oncogenic process of $\mathrm{OC}$ and follicular tumors is different and that other molecular mutations remain to be discovered. In view of the similarities between OC and FC with regard to expression of differentiated function and splicing anomalies of tpo, it can be assumed that such mutations have overlapping pathways.

ICC of TPO using MoAb47 was the first marker procedure proposed to assist diagnosis of malignancy on thyroid FNA (De Micco et al. 1994a). Its value has been confirmed by several independent studies with sensitivity and specificity up to $97.4 \%$ and 82\% respectively (De Micco et al. 1994a, 1999, Faroux et al. 1997, Christensen et al. 2000). To our knowledge, no other malignancy marker investigated in a large series of patients has presented sensitivity over $92 \%$ (Saggiorato et al. 2005). This quantitative analysis of tpo mRNA confirmed impairment of TPO expression in all histological thyroid carcinoma subtypes. In addition, we demonstrated that disruption of tpo mRNA maturation in follicular malignant thyroid tumors leads to overproduction of shorter TPO isoforms with different biological properties. This finding not only provides a plausible explanation for the TPO immunostaining anomalies observed using MoAb47 antibody in carcinomas but also lends further support to the diagnostic value of this marker in thyroid FNA.

\section{Funding}

This work was funded by a clinical research grant from the Public Hospital System of Marseille. The authors declare that there is no conflict of interest that would prejudice the impartiality of this scientific work.

\section{References}

Chazenbalk GD, Costante G, Portolano S, McLachlan SM \& Rapoport B 1993 The immunodominant region on human thyroid peroxidase recognized by autoantibodies does not contain the monoclonal antibody 47/c21 linear epitope. Journal of Clinical Endocrinology and Metabolism 77 1715-1718. 
Christensen L, Blichert-Toft M, Brandt M, Lange M, Sneppen SB, Ravnsbaek J, Mollerup CL, Strange L, Jensen F, Kirkegaard J, Hansen HS, Sorensen SS \& Feldt-Rasmussen UF 2000 Thyroperoxidase (TPO) immunostaining of the solitary cold thyroid nodule. Clinical Endocrinology 53 161-169.

Cohen Y, Xing M, Mambo E, Guo Z, Wu G, Trink B, Beller U, Westra WH, Ladenson PW \& Sidransky D 2003 BRAF mutation in papillary thyroid carcinoma. Journal of the National Cancer Institute 95 625-627.

Czarnocka B, Pastuszko D, Janota-Bzowski M, Weetman AP, Watson PF, Kemp EH, McIntosh RS, Asghar MS, Jarzab B, Gubala E, Wloch J \& Lange D 2001 Is there loss or qualitative changes in the expression of thyroid peroxidase protein in thyroid epithelial cancer? British Journal of Cancer 85 875-880.

De Lellis RA, Lloyd RV, Heitz PU \& Eng C 2004 World Health Organization classification of tumours. In Pathology and Genetics of Tumours of Endocrine Organs. pp 49-133 Lyon: IARC Press.

De Micco C, Ruf J, Chrestian MA, Gros N, Henry JF \& Carayon P 1991 Immunohistochemical study of thyroid peroxidase in normal, hyperplastic, and neoplastic human thyroid tissues. Cancer 67 3036-3041.

De Micco C, Zoro P, Garcia S, Skoog L, Tani EM, Carayon P \& Henry JF 1994a Thyroid peroxidase immunodetection as a tool to assist diagnosis of thyroid nodules on fine-needle aspiration biopsy. European Journal of Endocrinology 131 474-479.

De Micco C, Vasko V, Garcia S, Zoro P, Denizot A \& Henry JF $1994 b$ Fine-needle aspiration of thyroid follicular neoplasm: diagnostic use of thyroid peroxidase immunocytochemistry with monoclonal antibody 47. Surgery 116 1031-1035.

De Micco C, Vassko V \& Henry JF 1999 The value of thyroid peroxidase immunohistochemistry for preoperative fine-needle aspiration diagnosis of the follicular variant of papillary thyroid cancer. Surgery 126 1200-1204.

De Micco C, Kopp F, Vassko V \& Grino M 2000 In situ hybridization and immunohistochemistry study of thyroid peroxidase expression in thyroid tumors. Thyroid $\mathbf{1 0}$ $109-115$.

Di Cristofaro J, Vasko V, Savchenko V, Cherenko S, Larin A, Ringel MD, Saji M, Marcy M, Henry JF, Carayon P \& De Micco C 2005 ret/PTC1 and ret/PTC3 in thyroid tumors from Chernobyl liquidators: comparison with sporadic tumors from Ukrainian and French patients.

Endocrine-Related Cancer 12 173-183.

Dwight T, Thoppe SR, Foukakis T, Lui WO, Wallin G, Hoog A, Frisk T, Larsson C \& Zedenius J 2003 Involvement of the PAX8/peroxisome proliferator-activated receptor gamma rearrangement in follicular thyroid tumors. Journal of Clinical Endocrinology and Metabolism $\mathbf{8 8}$ 4440-4445.

Elisei R, Vassart G \& Ludgate M 1991 Demonstration of the existence of the alternatively spliced form of thyroid peroxidase in normal thyroid. Journal of Clinical Endocrinology and Metabolism 72 700-702.
Elisei R, Pinchera A, Romei C, Gryczynska M, Pohl V, Maenhaut C, Fugazzola L \& Pacini F 1994 Expression of thyrotropin receptor (TSH-R), thyroglobulin, thyroperoxidase, and calcitonin messenger ribonucleic acids in thyroid carcinomas: evidence of TSH-R gene transcript in medullary histotype. Journal of Clinical Endocrinology and Metabolism 78 867-871.

Fabbro D, Di Loreto C, Beltrami CA, Belfiore A, Di Lauro R, Damante G 1994 Expression of thyroid-specific transcription factors TTF-1 and PAX-8 in human thyroid neoplasms. Cancer Research 54 4744-4749.

Faroux MJ, Theobald S, Pluot M, Patey M \& Menzies D 1997 Evaluation of the monoclonal antibody antithyroperoxidase MoAb47 in the diagnostic decision of cold thyroid nodules by fine-needle aspiration. Pathology Research and Practice 193 705-712.

Fayadat L, Niccoli-Sire P, Lanet J \& Franc JL 1998 Human thyroperoxidase is largely retained and rapidly degraded in the endoplasmic reticulum. Its $\mathrm{N}$-glycans are required for folding and intracellular trafficking. Endocrinology 139 4277-4285.

Ferrand M, Franc JL, Henry JF \& De Micco C 2001 Overexpression of splicing variants of thyroperoxidase (TPO) in malignant thyroid tumours: correlation to alterations of TPO immunostaining. Journal of Endocrinological Investigations 24 (Suppl 6) 27.

Ferrand M, Le Fourn V \& Franc JL 2003 Increasing diversity of human thyroperoxidase generated by alternative splicing. Characterized by molecular cloning of new transcripts with single- and multispliced mRNAs. Journal of Biological Chemistry 278 3793-3800.

Gire V \& Wynford-Thomas D 2000 RAS oncogene activation induces proliferation in normal human thyroid epithelial cells without loss of differentiation. Oncogene 19 737-744.

Hoang-Vu C, Dralle H, Scheumann G, Maenhaut C, Horn R, von zur Muhlen A \& Brabant G 1992 Gene expression of differentiation- and dedifferentiation markers in normal and malignant human thyroid tissues. Experimental and Clinical Endocrinology 100 51-56.

Huang Y, Prasad M, Lemon WJ, Hampel H, Wright FA, Kornacker K, LiVolsi V, Frankel W, Kloos RT, Eng C, Pellegata NS \& de La Chapelle 2001 Gene expression in papillary thyroid carcinoma reveals highly consistent profiles. PNAS 98 15044-15049.

Kim KH, Kang DW, Kim SH, Seong IO \& Kang DY 2004 Mutations of the BRAF gene in papillary thyroid carcinoma in a Korean population. Yonsei Medical Journal 45 818-821.

Kimura S, Kotani T, McBride OW, Umeki K, Hirai K, Nakayama T \& Ohtaki S 1987 Human thyroid peroxidase: complete cDNA and protein sequence, chromosome mapping, and identification of two alternately spliced mRNAs. PNAS 84 5555-5559.

Lazar V, Bidart JM, Caillou B, Mahe C, Lacroix L, Filetti S \& Schlumberger M 1999 Expression of the $\mathrm{Na}+/ \mathrm{I}-$ symporter gene in human thyroid tumors: a comparison study with other thyroid-specific genes. Journal of Clinical Endocrinology and Metabolism 84 3228-3234. 
Le Fourn V, Ferrand M \& Franc JL 2004 Differential expression of thyroperoxidase mRNA splice variants in human thyroid tumors. Biochimica et Biophysica Acta 1689 134-141.

Lui WO, Foukakis T, Liden J, Thoppe SR, Dwight T, Hoog A, Zedenius J, Wallin G, Reimers M \& Larsson C 2005 Expression profiling reveals a distinct transcription signature in follicular thyroid carcinomas with a PAX8-PPAR(gamma) fusion oncogene. Oncogene 24 1467-1476.

Melillo RM, Castellone MD, Guarino V, De Falco V, Cirafici AM, Salvatore G, Caiazzo F, Basolo F, Giannini R, Kruhoffer M, Orntoft T, Fusco A \& Santoro M. 2005 The RET/PTC-RAS-BRAF linear signaling cascade mediates the motile and mitogenic phenotype of thyroid cancer cells. Journal of Clinical Investigation 115 1068-1081.

Mizukami Y, Nonomura A, Michigishi T, Noguchi M, Nakamura S, Arai Y, Kotani T, Ohtaki S \& Matsukawa S 1994 Immunohistochemical demonstration of thyroid peroxidase (TPO) in human thyroid tissues from various thyroid diseases. Anticancer Research 14 1329-1334.

Niccoli P, Fayadat L, Panneels V, Lanet J \& Franc JL 1997 Human thyroperoxidase in its alternatively spliced form (TPO2) is enzymatically inactive and exhibits changes in intracellular processing and trafficking. Journal of Biological Chemistry 272 29487-29492.

Niccoli-Sire P, Fayadat L, Siffroi-Fernandez S, Malthierry Y \& Franc JL 2001 Alternatively spliced form of human thyroperoxidase, TPOzanelli: activity, intracellular trafficking, and role in hormonogenesis. Biochemistry $\mathbf{4 0}$ 2572-2579.

Nikiforova MN, Biddinger PW, Caudill CM, Kroll TG \& Nikiforov YE 2002 PAX8-PPARgamma rearrangement in thyroid tumors: RT-PCR and immunohistochemical analyses. American Journal of Surgery and Pathology 26 1016-1023.

Nikiforova MN, Lynch RA, Biddinger PW, Alexander EK, Dorn GW 2nd, Tallini G, Kroll TG \& Nikiforov YE 2003 RAS point mutations and PAX8-PPAR gamma rearrangement in thyroid tumors: evidence for distinct molecular pathways in thyroid follicular carcinoma. Journal of Clinical Endocrinology and Metabolism $\mathbf{8 8}$ 2318-2326.

Nikiforova MN, Ciampi R, Salvatore G, Santoro M, Gandhi M, Knauf JA, Thomas GA, Jeremiah S, Bogdanova TI, Tronko MD, Fagin JA \& Nikiforov YE 2004 Low prevalence of BRAF mutations in radiation-induced thyroid tumors in contrast to sporadic papillary carcinomas. Cancer Letters 209 1-6.

Picard C, Silvy M \& Gabert J 2006 Overview of real-time RT-PCR strategies for quantification of gene rearrangements in the myeloid malignancies. Methods in Molecular Medicine 125 27-68.

Pohl V, Abramowicz M, Vassart G, Dumont JE \& Roger PP 1993 Thyroperoxidase mRNA in quiescent and proliferating thyroid epithelial cells: expression and subcellular localization studied by in situ hybridization. European Journal of Cell Biology 62 94-104.

Saggiorato E, De Pompa R, Volante M, Cappia S, Arecco F, Dei Tos AP, Orlandi F \& Papotti M 2005 Characterization of thyroid 'follicular neoplasms' in fine-needle aspiration cytological specimens using a panel of immunohistochemical markers: a proposal for clinical application. Endocrine-Related Cancer 12 305-317.

Santoro M, Thomas GA, Vecchio G, Williams GH, Fusco A, Chiappetta G, Pozcharskaya V, Bogdanova TI,

Demidchik EP, Cherstvoy ED, Voscoboinik L, Tronko ND, Carss A, Bunnell H, Tonnachera M, Parma J, Dumont JE, Keller G, Hofler H \& Williams ED 2000 Gene rearrangement and Chernobyl related thyroid cancers. British Journal of Cancer 82 315-322.

Sarquis MS, Weber F, Shen L, Broelsch CE, Jhiang SM, Zedenius J, Frilling A \& Eng C 2006 High frequency of loss of heterozygosity in imprinted, compared with nonimprinted, genomic regions in follicular thyroid carcinomas and atypical adenomas. Journal of Clinical Endocrinology and Metabolism 91 262-269.

Tanaka T, Umeki K, Yamamoto I, Sugiyama S, Noguchi S \& Ohtaki S 1996 Immunohistochemical loss of thyroid peroxidase in papillary thyroid carcinoma: strong suppression of peroxidase gene expression. Journal of Pathology 179 89-94.

Trovisco V, Vieira de Castro I, Soares P, Maximo V, Silva P, Magalhaes J, Abrosimov A, Guiu XM \& Sobrinho-Simoes M 2004 BRAF mutations are associated with some histological types of papillary thyroid carcinoma. Journal of Pathology 202 247-251.

Vandenbroucke II, Vandesompele J, Paepe AD \& Messiaen L 2001 Quantification of splice variants using real-time PCR. Nucleic Acids Research 29 E68.

Vasko V, Ferrand M, Di Cristofaro J, Carayon P, Henry JF \& de Micco C 2003 Specific pattern of RAS oncogene mutations in follicular thyroid tumors. Journal of Clinical Endocrinology and Metabolism 88 2745-2752.

Vasko VV, Gaudart J, Allasia C, Savchenko V, Di Cristofaro J, Saji M, Ringel MD \& De Micco C 2004 Thyroid follicular adenomas may display features of follicular carcinoma and follicular variant of papillary carcinoma. European Journal of Endocrinology 151 779-786.

Xing M 2005 BRAF mutation in thyroid cancer. Endocrine-Related Cancer 12 245-262.

Xing M, Vasko V, Tallini G, Larin A, Wu G, Udelsman R, Ringel MD, Ladenson PW \& Sidransky D 2004 BRAF T1796A transversion mutation in various thyroid neoplasms. Journal of Clinical Endocrinology and Metabolism 89 1365-1368.

Zanelli E, Henry M, Charvet B \& Malthiery Y 1990 Evidence for an alternate splicing in the thyroperoxidase messenger from patients with Graves' disease. Biochemical and Biophysical Research Communications 170 735-741. 
